\title{
mir-35 is involved in intestine cell G1/S transition and germ cell proliferation in C. elegans
}

\author{
Min Liu ${ }^{1, *}$, Pengpeng Liu ${ }^{1, *}$, Li Zhang ${ }^{2}$, Qingchun Cai ${ }^{1,3}, \mathrm{Ge} \mathrm{Gao}^{2}$, Wenxia Zhang ${ }^{1}$, Zuoyan Zhu, Dong Liu ${ }^{1}$ \\ Qichang Fan
}

${ }^{I}$ The Education Ministry Key Laboratory of Cell Proliferation and Differentiation and the State Key Laboratory of Bio-membrane and Membrane Bio-engineering; ${ }^{2}$ The Center for Bioinformatics, School of Life Sciences, Peking University, 5 Summer Palace Road, Beijing 100871, China

MicroRNA (miRNA) regulates gene expression in many cellular events, yet functions of only a few miRNAs are known in $C$. elegans. We analyzed the function of mir-35-41 unique to the worm, and show here that mir-35 regulates the G1/S transition of intestinal cells and germ cell proliferation. Loss of mir-35 leads to a decrease of nuclei numbers in intestine and distal mitotic gonad, while re-introduction of mir-35 rescues the mutant phenotypes. Genetic analysis indicates that mir-35 may act through $\mathrm{Rb} / \mathrm{E} 2 \mathrm{~F}$ and SCF pathways. Further bioinformatic and functional analyses demonstrate that mir-35 targets evolutionally conserved lin-23 and gld-1. Together, our study reveals a novel function of mir-35 family in cell division regulation.

Keywords: miRNA; C. elegans; mir-35; G1/S transition; germ cell proliferation

Cell Research (2011) 21:1605-1618. doi:10.1038/cr.2011.102; published online 21 June 2011

\section{Introduction}

miRNAs are non-coding transcripts of 19-25 nucleotides in eukaryotes. Since lin-4 was first identified in C. elegans [1], hundreds of miRNA species, with important functions in a variety of biological processes, have been identified in various multi-cell organisms. These small RNAs regulate gene expression mainly through degradation of target mRNAs [2], termination of translation [3], and chromatin modification [4]. In $C$. elegans, there are currently about 170 miRNA genes listed in the miRBase sequence database (http://

\footnotetext{
*These two authors contributed equally to this work.

Correspondence: Dong Liu ${ }^{\mathrm{a}}$, Qichang Fan ${ }^{\mathrm{b}}$

${ }^{a}$ E-mail: doliu@pku.edu.cn

${ }^{\mathrm{b}}$ E-mail: qfan@pku.edu.cn

${ }^{3}$ Current address: Department of Obstetrics and Gynecology, Indiana University School of Medicine Indianapolis, Indianapolis, IN 46202, USA Abbreviations: CDC (Cell Division Cycle related), CKI (Cyclin-dependent Kinase Inhibitor), CUL (CULin), CYE (CYclin E), DPL (DPLike protein), EFL (E2F-like protein), GLP (abnormal Germ Line Proliferation), GLD (defective in Germ Line Development), LIN (abnormal cell LINeage), mir35 (microRNA-35), RB (Retinoblastoma protein), SCF (Skp1-Cul1-F box) Received 20 September 2010; revised 16 January 2011; accepted 10 February 2011; published online 21 June 2011
}

www.mirbase.org); however, only a few have been studied in detail [5-14]. Because deleting most miRNAs individually does not lead to any overt phenotypes/ abnormalities in development and differentiation [15], it has been a popular accord that functional redundancy of different miRNAs (miRNAs share the same or have diverse and overlapping target mRNAs) may contribute to the limited progress of miRNA study in the worm. The complexity of functional redundancy of miRNAs may further be contributed by tandem-arrayed miRNA gene clusters in the worm genome.

Because miRNAs normally function in a subtle way to safeguard or buffer developmental or physiological processes, and/or to coordinate multiple gene regulatory pathways, functions of individual miRNAs could hardly be distinguishable in normal conditions. Recent miRNA studies suggest that previously unknown miRNA functions may be revealed under sensitized or stressed conditions [16]. For instance, mir-1 has been indicated in modulating synaptic function when neuro-musculature signaling is compromised [17]. Recently, Brenner et al. reported that in sensitized situations, either genetically or environmentally, critical roles of several known miRNAs could be revealed, indicating that a group of seemingly unrelated miRNAs works redundantly on distinct target 
mRNAs of parallel regulatory genetic pathways, and/or on the very same targets that integrate distinct genetic pathways in a group of specialized cells [16]. Therefore, against genetic and/or environmental changes, micro effects of individual miRNAs would be amplified.

The mir-35 family (mir-35-41 cluster and mir-42) and its expression pattern were first described in 2001 [14]. Later in a genome-wide analysis of miRNA promoter activities, the pri-mir-35-41 was found ubiquitously expressed in embryo and all larva stages [18]. Early embryonic death was evident when the entire cluster, including mir-42, was deleted [14]; however, the genetic basis of how the mir-35 family affects post-embryonic worm development was not explored. We sought to study the function of mir-35 family in a temperature sensitive mutant, gk262, in which only the mir-35-41 cluster was deleted (Figure 1A). Combining genetics and other approaches, we discovered that mir-35 functions in G1/
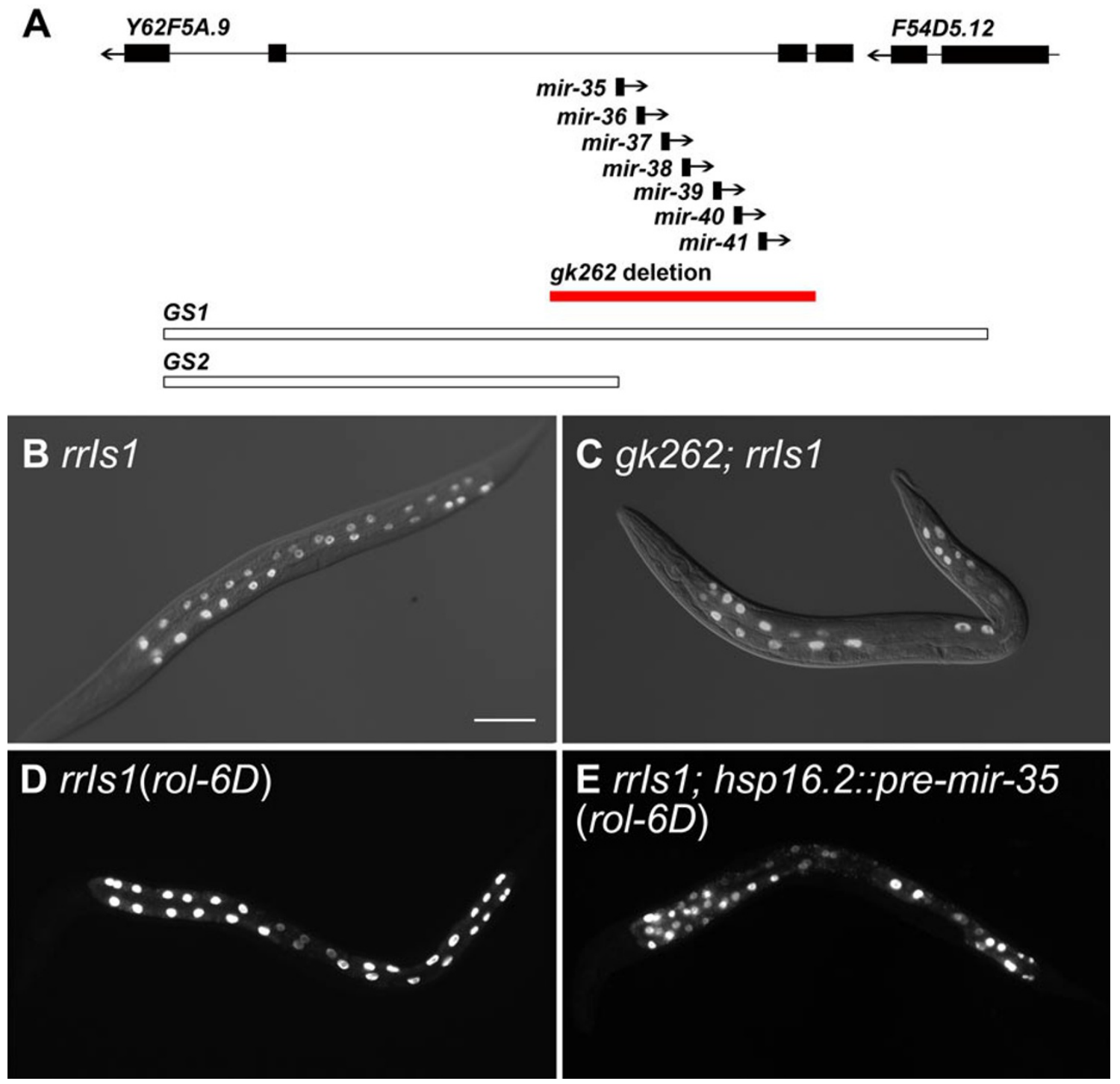

Figure 1 mir-35 affects the cell cycle control in intestinal cells. (A) The schematic genomic location of mir-35-41 cluster. The mutant gk262 has a 1268 bp deletion at Y62F5A.9 locus, removing the whole mir-35-41 cluster. GS1 is a 4.06 kb-fragment that covers the entire mir-35-41 locus. GS2 is a 2.16-kb fragment upstream of mir-35-41 cluster locus, which includes the promoter of mir-35. (B) The rrls1[elt-2::GFP] marker visualizes the nuclei of the intestine cells. (C) gk262 animals exhibit a decrease in the intestine nuclei number, (D, E) mir-35 over-expression increases the intestine nuclei number. (D) rrls1 carrying a rol-6D co-microinjection marker as a control. (F) The transgenic hsp-16.2::pre-mir-35(rol-6D) animals show a increase in the intestine nuclei number. The scale bar represents $100 \mu \mathrm{m}$. 
Table 1 Number of intestinal nuclei in $g k 262$ mutants

\begin{tabular}{|c|c|c|c|c|}
\hline & \multicolumn{2}{|l|}{$20{ }^{\circ} \mathrm{C}$} & \multicolumn{2}{|c|}{$25^{\circ} \mathrm{C}$ (temperature shift) } \\
\hline & rrIs1 & $g k 262 ; r r I s 1$ & $r r I s 1$ & $g k 262 ; r r I s 1$ \\
\hline 3-fold stage & $19.0 \pm 0.6(49)^{*}, 18-20^{* *}$ & $17.0 \pm 2.3(45), 11-20$ & $18.8 \pm 1.0(56), 17-20$ & $16.0 \pm 2.3(48), 11-20$ \\
\hline L3/L4 stage & $31.0 \pm 1.4(83), 27-34$ & $26.3 \pm 3.2(141), 19-34$ & $30.7 \pm 1.6(93), 26-34$ & $22.5 \pm 3.4(102), 16-30$ \\
\hline
\end{tabular}

${ }^{*}$ The number in the bracket is the total animal number examined.

${ }^{* *}$ The dash indicates the range of the number.

\begin{tabular}{|c|c|c|}
\hline Strains & & Intestinal nuclei \\
\hline rol-6D & & $32.0 \pm 1.1(91)^{*}, 28-34^{* *}$ \\
\hline gk262; GS1(rol-6D) & & $32.0 \pm 2.0(46), 29-34$ \\
\hline \multirow[t]{2}{*}{ gk262; GS2::pre-mir-35(rol-6D) } & line1 & $31.6 \pm 2.1(51), 30-35$ \\
\hline & line2 & $31.5 \pm 2.9(47), 29-34$ \\
\hline \multirow[t]{2}{*}{ gk262; GS2::pre-mir-36(rol-6D) } & line1 & $32.1 \pm 1.8(43), 29-35$ \\
\hline & line2 & $31.2 \pm 1.3(39), 29-34$ \\
\hline \multirow[t]{2}{*}{ gk262; GS2::pre-mir-37(rol-6D) } & line1 & $32.0 \pm 2.0(50), 28-38$ \\
\hline & line2 & $31.5 \pm 1.7(33), 28-35$ \\
\hline \multirow[t]{2}{*}{ gk262; GS2::pre-mir-38(rol-6D) } & line1 & $32.6 \pm 1.5(38), 29-35$ \\
\hline & line2 & $31.7 \pm 1.7(31), 28-35$ \\
\hline \multirow[t]{2}{*}{ gk262; GS2::pre-mir-39(rol-6D) } & line1 & $31.5 \pm 1.6(48), 27-35$ \\
\hline & line2 & $30.8 \pm 1.4(34), 28-34$ \\
\hline \multirow[t]{2}{*}{ gk262; GS2::pre-mir-40(rol-6D) } & line1 & $31.6 \pm 1.7(37), 27-35$ \\
\hline & line2 & $31.0 \pm 1.5(42), 28-35$ \\
\hline \multirow[t]{2}{*}{ gk262; GS2::pre-mir-41(rol-6D) } & line1 & $31.1 \pm 1.6(55), 27-34$ \\
\hline & line2 & $30.7 \pm 1.5(34), 27-34$ \\
\hline \multirow[t]{2}{*}{ gk262; ges-1::pre-mir-35(rol-6D) } & line1 & $33.5 \pm 1.6(49), 30-38$ \\
\hline & line2 & $33.3 \pm 1.7(50), 29-38$ \\
\hline \multirow[t]{3}{*}{ hsp16-2::pre-mir-35(rol-6D) $\left(20^{\circ} \mathrm{C}\right)$} & line1 & $35.6 \pm 2.0(55), 32-41$ \\
\hline & line2 & $35.3 \pm 1.0(67), 33-37$ \\
\hline & line3 & $35.4 \pm 1.4(81), 32-39$ \\
\hline
\end{tabular}

"The number in the bracket is the total animal number examined.

${ }^{* *}$ The dash indicates the range of the number.

$\mathrm{S}$ transition to regulate intestinal cell cycle and germ cell proliferation.

G1/S transition is governed by three identified pathways, CKI (Cyclin-dependent Kinase Inhibitor), SCF (Skp1-Cul1-F box) and Rb (Retinoblastoma protein)/E2F. We find that mir-35 participates in G1/S transition mainly through inhibiting $\mathrm{Rb} / \mathrm{E} 2 \mathrm{~F}$ and $\mathrm{SCF}$ pathways during intestine development. In the gonad, GLP-1 (abnormal Germ Line Proliferation)/Notch signaling is essential to promote germ line divisions in distal mitotic region [19-22]. GLD (defective in Germ Line Development) family plays a key role in determining meiosis entry and indirectly regulates mitotic proliferation of the germline [23-27]. We found that mir-35 may down-regulate gld-1 to ensure a normal germline proliferation in distal mitotic region. Together, our study reveals that mir-35 coordinates distinct signaling/regulatory pathways in cell cycle and proliferation.

\section{Results}

Lacking mir-35 causes decrease of intestinal nuclei numbers

In C. elegans, wild-type embryo has 20 intestine cells, each of which has a diploid nucleus at 3-fold stage [28]. After hatching, some intestinal nuclei replicate without cell division, thus 30 to 34 diploid nuclei are present in the 20 intestine cells since late L1 stage [28]. Transgenic GFP line $r r I s 1[$ elt-2::GFP] specifically labels intestine nuclei (Figure 1B). When rrIs 1 was introduced into 


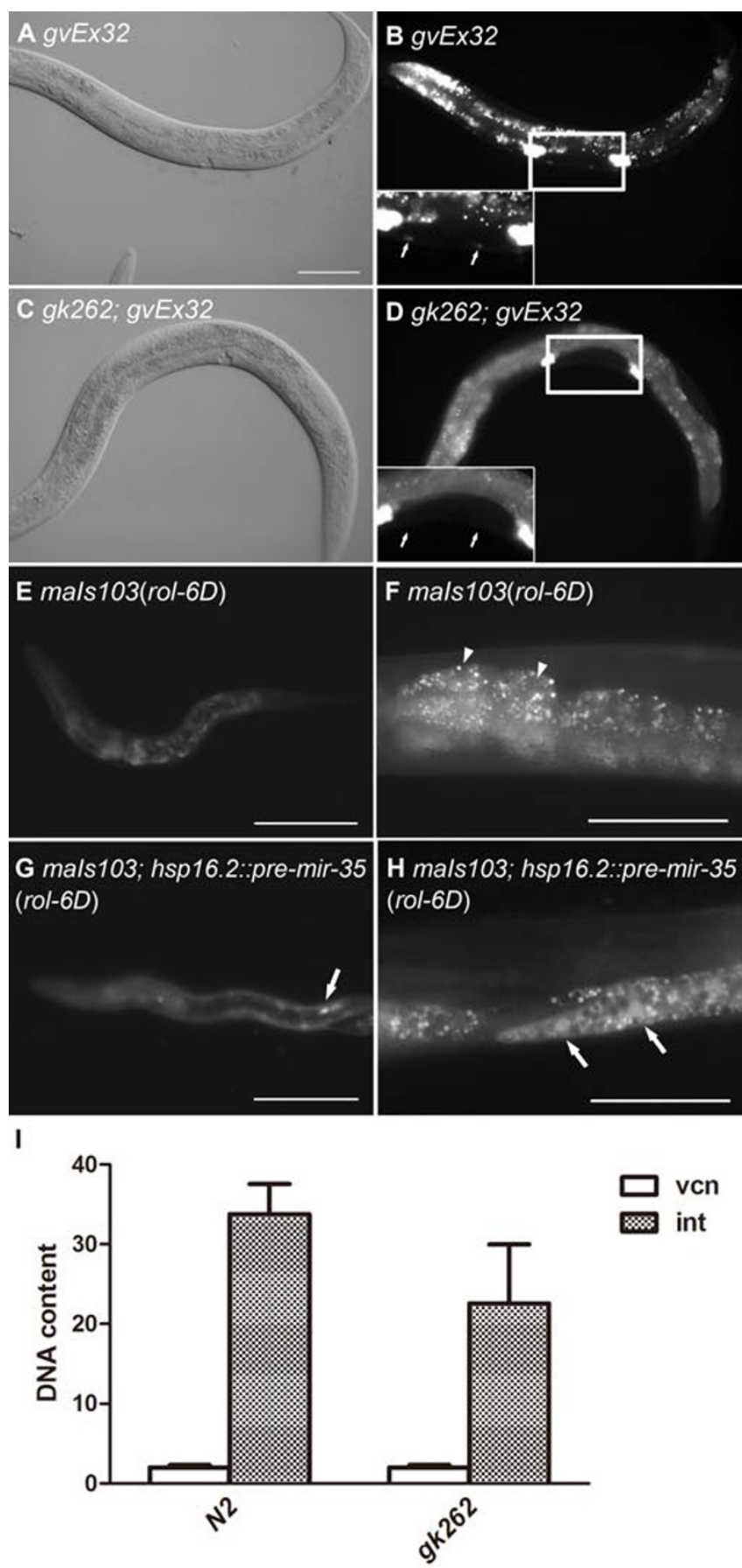

the mir-35 mutant $g k 262$, less intestinal nuclei were observed than the wide type, at 3-fold and L3/L4 stages, respectively (Table 1, $P<0.001$, Student $t$-test). As a temperature sensitive deficiency, $g k 262$ is embryonic lethal at $25{ }^{\circ} \mathrm{C}$. Using temperature shift assay (i.e., synchronized young adults were allowed to lay eggs for $1 \mathrm{~h}$ at $20^{\circ} \mathrm{C}$, and the eggs were shifted to and maintained at $25^{\circ} \mathrm{C}$ ), we found $g k 262$ survivors had only an average
Figure 2 mir-35 affects DNA replication. (A, B) The gvEx32 [cye-1::GFP; rol-6D] marker visualizes the nuclei in the process of DNA replication. The DIC (A) and the cye-1::GFP (B) picture of the same animal at early L4 stage. In the enlarged region, the arrows indicate two hypodermal cell nuclei that are replicating DNA. (C, D) gk262; gvEx32 shows less GFP-expressing cells after the temperature shift. The DIC (C) and the cye-1::GFP (D) picture of the same animal at the same stage as shown in $\mathbf{A}$ and $\mathbf{B}$. In the enlarged region, the arrows indicate two hypodermal cell nuclei that lose DNA replication. (E, F) The mals103[rnr::GFP] marker also visualizes the nuclei in the process of DNA replication. While at the arrest $L 1(E)$ and adult (F) stage, all the somatic cell nuclei stop DNA replication. The arrowheads in $\mathrm{F}$ indicate intestinal nuclei, which stop DNA replication. (G, H) mals103 carrying a hsp-16.2::pre-mir-35 transgene could induce DNA replication in the intestinal nuclei at the arrest L1 (G) and adult (H) stage after heat-shock. The arrows in $\mathbf{G}$ and $\mathbf{H}$ indicate intestinal nuclei in which DNA is synthesized. The scale bars represent $100 \mu \mathrm{m}$. (I) Intestinal ploidy measurement in N2 and gk262. Ventral cord neuron nuclei were used as an internal $2 \mathrm{n}$ standard. White bar indicates the average DNA content of over 50 ventral cord nuclei in 10 independent animals. Black bar indicates the average DNA content of over 50 intestinal nuclei in 10 independent animals. Error bars represent standard deviation (SD).

of $16.0(n=48)$ and $22.5(n=102)$ intestinal nuclei at 3-fold and L3/L4 stages, respectively, significantly less than the wide type (Figure 1C, Table $1, P<0.001$, Student $t$-test). The DAPI (4',6-diamidino-2-phenylindole dihydrochloride) nuclear staining also indicated such a decrease (data not shown). Therefore, intestinal E lineage is abnormal in $g k 262$.

gk262 has a 1268 bp deletion at the Y62F5A.9 locus, removing the gene's entire 2 nd exon and portions of 1 st and 2nd introns. mir-35-41 cluster resides in an opposite orientation of $Y 62 F 5 \mathrm{~A} .9$ and is completely missing in gk262 (Figure 1A). A $4.06 \mathrm{~kb}-\mathrm{PCR}$ product (GS1) that covers the entire mir-35-41 locus and partial Y62F5A.9 (Figure 1A), could fully rescue embryonic lethality and intestinal defect of $g k 262$ at $25{ }^{\circ} \mathrm{C}$, i.e., there were 32.0 $\pm 2.0^{\circ} \mathrm{C}(n=46)$ intestinal nuclei in transgenic $g k 262$; GS1. We also used a 2.16-kb fragment (GS2, Figure 1A) to drive the expression of each of the seven members of the mir-35 cluster, and every resulted construct completely rescued $g k 262$ intestine defects at $25^{\circ} \mathrm{C}$ (Table 2 ). When pre-mir-35 was driven by an intestine specific ges-1 promoter and expressed in $g k 262$, the intestinaldefects could be fully rescued (Table 2). In fact, excess intestinal nuclei could also be observed in the transgenic gk262 (Table 2). When we mis-expressed either premir-35 or pre-mir-35-41 with a heat-shock promoter, we found that the intestinal nuclei number was further 
Table 3 Number of intestinal nuclei in the double mutants of $g k 262$ and G1/S related genes at L3/L4 stage

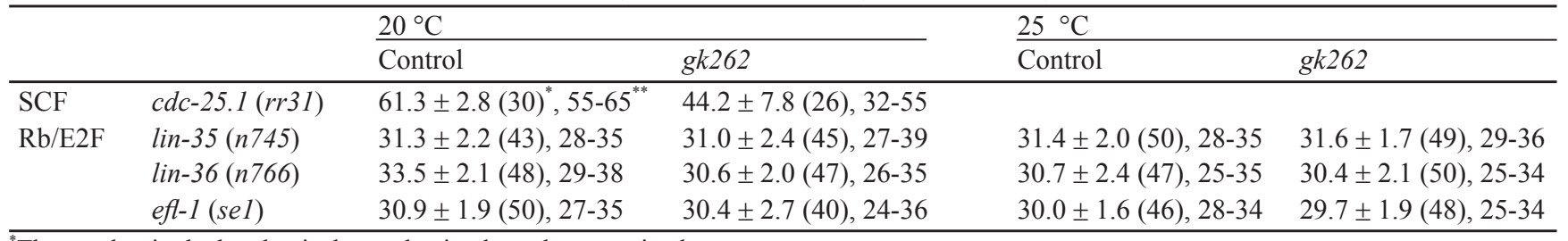

${ }^{*}$ The number in the bracket is the total animal number examined.

${ }^{* *}$ The dash indicates the range of the number.

Table 4 Number of intestinal nuclei in the double mutants of $g k 262$ and G1/S related genes at 3-fold stage

\begin{tabular}{|c|c|c|c|c|c|}
\hline & & \multicolumn{2}{|l|}{$20^{\circ} \mathrm{C}$} & \multicolumn{2}{|l|}{$25^{\circ} \mathrm{C}$} \\
\hline & & Control & $g k 262$ & Control & $g k 262$ \\
\hline Inhibitor & $c k i-1(R N A i)$ & $28.8 \pm 1.7(31)^{*}, 26-32^{* *}$ & $20.9 \pm 2.0(18), 16-24$ & $29.4 \pm 1.4(54), 25-32$ & $21.5 \pm 2.4(48), 15-28$ \\
\hline \multirow[t]{3}{*}{$\mathrm{Rb} / \mathrm{E} 2 \mathrm{~F}$} & $d p l-1(R N A i)$ & $22.9 \pm 2.3(55), 19-28$ & $16.7 \pm 2.0(43), 14-20$ & $22.6 \pm 2.7(72), 19-41$ & $15.7 \pm 2.1(57), 11-20$ \\
\hline & $\operatorname{lin}-9(R N A i)$ & $20.9 \pm 1.9(44), 19-23$ & $17.9 \pm 1.8(71), 14-22$ & $20.4 \pm 1.1(56), 18-22$ & $17.4 \pm 1.4(81), 14-22$ \\
\hline & $\operatorname{lin}-15 B(R N A i)$ & $21.0 \pm 1.7(39), 18-23$ & $19.1 \pm 1.7(31), 13-21$ & $20.2 \pm 1.2(58), 17-23$ & $18.1 \pm 1.7(54), 12-21$ \\
\hline
\end{tabular}

${ }^{*}$ The number in the bracket is the total animal number examined.

${ }^{* *}$ The dash indicates the range of the number.

increased (Figure 1D-1E; Table 2, $P<0.001$, Student $t$-test). These results indicate that the loss of the mir-3541 cluster is responsible for $g k 262$ intestine defects.

mir-35 affects DNA replication of intestine nuclei

In $C$. elegans, all intestinal nuclei endoreduplicate their DNA prior to each of four molts, thereby producing $32 \mathrm{n}$ nuclei in adult intestine cells [28]. DNA content of intestinal nuclei in adult $g k 262$ was measured. Using normally divided ventral cord neuron as 2 n control, partially visualized by transgenic GFP lines juIs 14 [acr$2: \because G F P]$ and juIs $76[$ unc-25::GFP] (data not shown), we found that DNA content of intestinal nuclei was $22.6 \pm$ $7.4(n=68)$ in $g k 262$, much less than that of $33.3 \pm 3.8$ $(n=52)$ in wild type (Figure 2I, $P<0.01$ Student $t$-test). Moreover, we followed nuclear DNA synthesis in $g k 262$ using two transgenic GFP lines, maIs $103[$ rnr::GFP] and gvEx32[cye-1::GFP; rol-6D], both of which specifically marked nuclei entering $\mathrm{S}$ phase (Figure 2A, 2B, 2E and $2 \mathrm{~F}$ ). We observed less GFP-expressing nuclei in $g k 262$; gvEx32 (Figure 2C and 2D). Furthermore, heat-induced ectopic expression of pre-mir-35 in L1 arrest and adult maIs 103 worms resulted in $r n r:: G F P$ expression in intestinal nuclei (Figure 2E-2H). These results indicate that mir-35 could trigger DNA synthesis in quiescent cells (i.e. cells in terminally differentiated state), thus regulating G1/S transition of the cell cycle in intestine cells. mir-35 engages in the Rb/E2F and SCF pathways in regulating $G 1 / S$ transition

To determine how mir-35 affects G1/S transition, we analyzed genetic interactions of mir-35 and pivotal genes in the three main regulatory pathways. CKI-1 was reported to negatively regulate G1/S transition [29-31] as one of the most important CKIs. In cki-1(RNAi), gk262, the number of intestinal nuclei was between those of cki-1(RNAi) and $g k 262$ at 3-fold stage (Tables 1 and 4), showing a partial suppression effect of $c k i-1$ on $g k 262$. Meanwhile, $c k i-1(R N A i) ; g k 262$ led to an additive effect on embryonic lethality. These data indicated that $c k i-1$ function may be mediated partially through mir-35.

The SCF complex functions as an E3 ubiquitin ligase and degrades candidate target proteins, including CYE-1 (cyclin E-1) [32, 33] and CDC-25.1 (Cell Division Cycle related) [34], to negatively regulate $\mathrm{G} 1 / \mathrm{S}$ transition. In C. elegans, cul-1 (CULlin) and lin-23 (abnormal cell LINeage) have been identified to encode main components of this complex [35, 36], and depletion of lin-23 leads to intestinal hyperplasia [34]. We found lin-23(RNAi); gk262 showed a high rate of embryonic lethality and lin-23(RNAi)-induced hyperplasia could hardly be restored in $g k 262$ (Tables 3 and 4), suggesting that lin-23 may function downstream of mir-35. We also examined the genetic interaction between $c d c-25.1$ and mir-35. Because known loss-of-function alleles of $c d c$ 25.1 do not show any intestine defect $[37,38]$, and $c d c$ - 
A

mir-35 3' UGACGAUCAAAGGUGGGCCAC '5 | $|1:||:||||||| \mid$ lin-23 5' TACTGATCTTCAACCGGTG'3
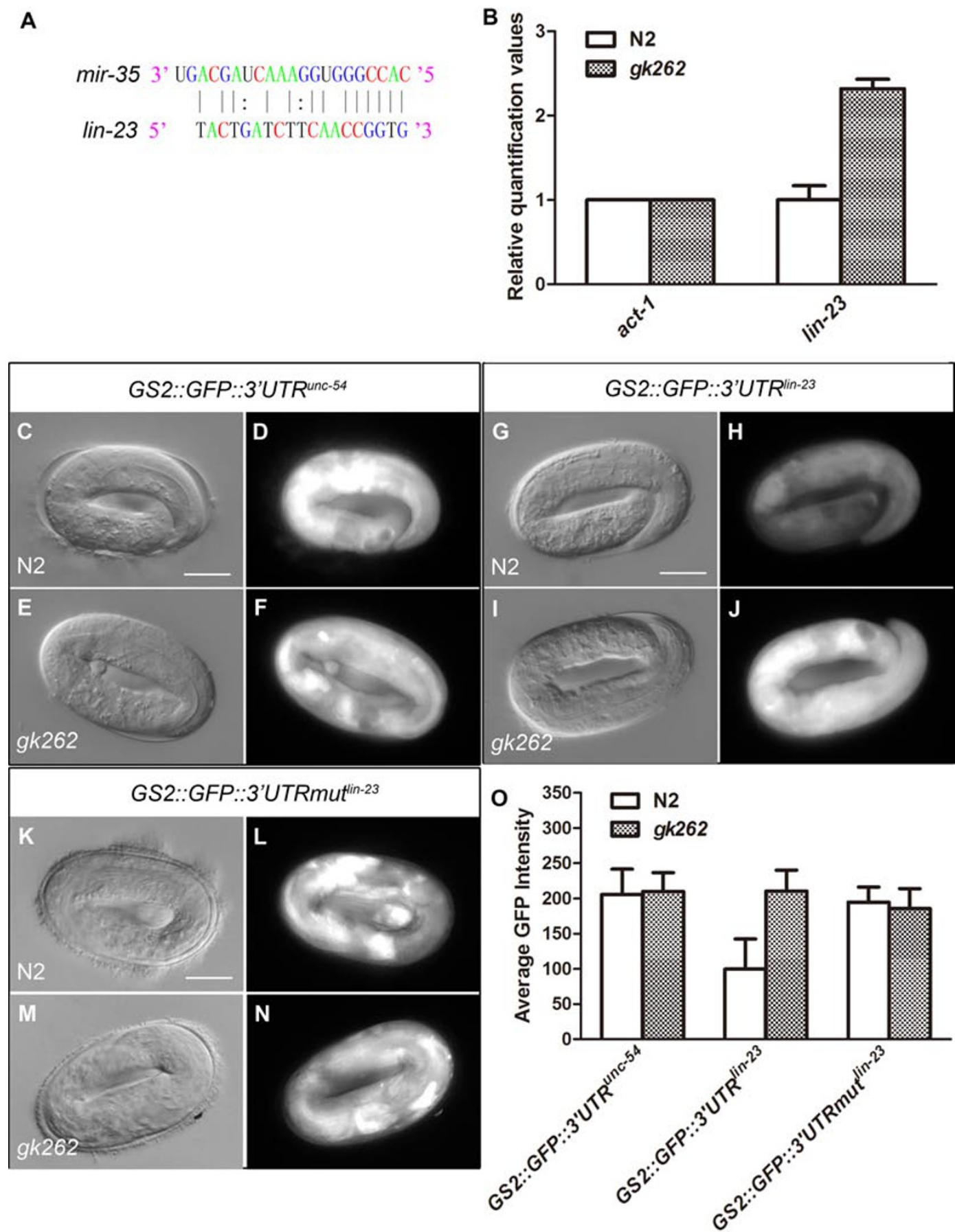

Figure 3 lin-23 may be a direct target of mir-35. (A) lin-23 is predicted as a target of mir-35 by the bioinformatics program miRanda. Solid lines indicate perfect match, while dash lines indicate non-perfect match. (B) The mRNA level of lin-23 is up regulated in the gk262 at young adult stage revealed by qRT-PCR. (C-N) in vivo GPF reporter experiment shows that lin-23 is a direct target of mir-35. (C-F) The 3' UTR of unc-54 has no binding site for mir-35, which is used as a control, no obvious difference of GFP level could be observed between wild type and gk262. The DIC (C, E) and the GFP (D, F) picture of the same embryo at 2-3 fold stage. (G-J) A much higher GFP level in gk262 was observed compared to that of the wild type. The DIC (C, E) and the GFP (D, F) pictures are of the same embryos at 2-3 fold stage. (K-N) The $3^{\prime} U T R m t^{\text {lin-23 }}$ has a mutated binding site for mir-35. No obvious difference of GFP level could be observed between wild type and gk262. The DIC (K, M) and the GFP (L, N) pictures are of the same embryos at 2-3 fold stage. The scale bar represents $25 \mu \mathrm{m}$. (O) Quantification of average GFP intensity of

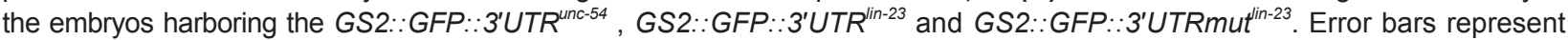
SD. 


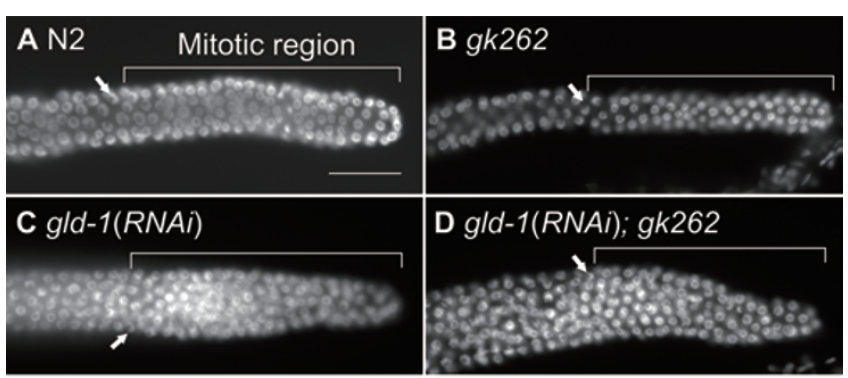

E

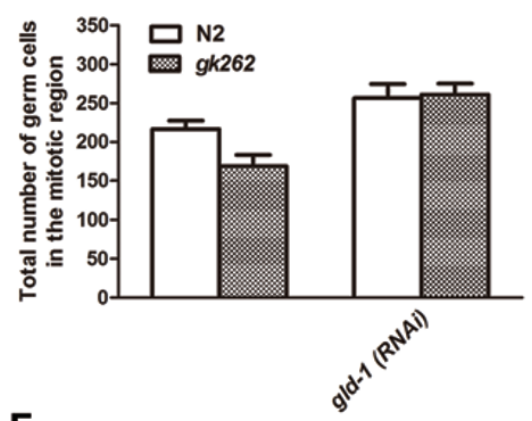

$\mathbf{F}$

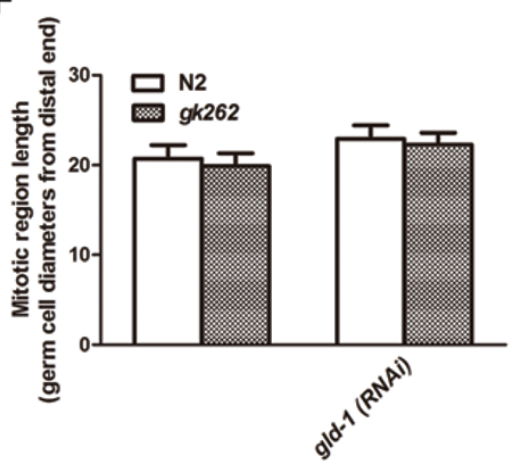

Figure 4 mir-35 affects adult germ line mitosis. (A-D) DAPI staining of distal ends of gonads, dissected from adult hermaphrodites. (B) gk262 mutant shows a thinner mitotic region than N2 (A), but the lengths of both are nearly the same. In (C) gld-1(RNAi) and (D) gld-1(RNAi); gk262, the mitotic regions overgrow, while the lengths of the mitotic regions are not changed. The arrows indicate the crest-like germ cells in the transition zones. The scale bar represents $50 \mu \mathrm{m}$. (E) Total number of germ cells in the mitotic region of N2 and gk262, and the progeny of animals performed gld-1(RNAi). Error bars represent SD. (F) Mitotic region length (germ cell diameters from distal end) of N2 and gk262, and the progeny of animals performed gld-1(RNAi). Error bars represent SD.

25.1RNAi-treated gk262 and lin-23(e1883) lines are early embryonic lethal (data not shown), we took advantage of a dominant gain-of-function $c d c-25.1$ mutant $r r 31$, which induces hyperplasia in the intestine [39]. Double mutant $r r 31 ; g k 262$ is early embryonic lethal at $25^{\circ} \mathrm{C}$. However, the $r r 31$; gk262 line could develop into fertile adulthood at $20^{\circ} \mathrm{C}$, while the hyperplasia in the intestine is suppressed (Tables 3 and 4), consistent with the fact that overexpression of Lin-23 reduced $c d c-25.1$ ( $r r 31$ )-dependent intestine hyperplasia [34].

The $\mathrm{Rb} / \mathrm{E} 2 \mathrm{~F}$ pathway include lin-35 Rb [40], lin-36 [41], dpl-1 (DP-Like) and efl-1 (E2F-Like) [42]. lin-35, lin-36 and efl-1 negatively regulate $\mathrm{S}$ phase entry [43, 44], while $d p l-1$ acts as both a positive and negative regulator $[44,45]$. We found that lin-35(n745), lin-36(n766) and efl1 (sel) could fully suppress intestinal nuclei phenotype of $g k 262$, at both $20^{\circ} \mathrm{C}$ and $25{ }^{\circ} \mathrm{C}$ (Tables 1 and 3, $P>$ 0.05 , Student $t$-test), indicating that mir-35 regulates G1/ $\mathrm{S}$ transition in an $\mathrm{Rb} / \mathrm{E} 2 \mathrm{~F}$-dependent manner.

lin-9 Mip130/TWIT and lin-15B were also identified as negative $\mathrm{G} 1$ regulatory proteins $[44,46]$, however, gk262; $\operatorname{lin}-9(n 112)$ and $g k 262$; $\operatorname{lin}-15 B(n 309)$ were embryonic lethals. lin-9(RNAi)- and lin-15B(RNAi)injected $g k 262$ showed limited restoration of intestine nuclei loss (Tables 3 and 4 ) at $25^{\circ} \mathrm{C}$, suggesting that lin-9 and $\operatorname{lin}-15 B$ may have no direct genetic interaction with mir-35. In summary, mir-35 inhibits the retinoblastoma protein/E2F and SCF pathways in regulating G1/S transition.

\section{mir-35 targets lin-23 to regulate G1/S transition}

lin-23 is a potential target of mir-35 (Figure 3A). Using qRT-PCR, we found that lin-23 gene expression level was up-regulated in $g k 262$ at $25{ }^{\circ} \mathrm{C}$ (Figure 3B). To further examine whether lin-23 is the target gene of mir-35, the 3' UTR of $l i n-23$ and GS2 (Figure 1A) were used to yield the GS2::GFP::3'UTR ${ }^{l i n-23}$ reporter DNA construct. A much higher GFP level in gk262, compared to that of the wild type (Figure 3G-3J and 3O, $n>50$, $P<0.001$, Student $t$-test) was observed. In contrast, the unc-54 3' UTR, which is lacking of mir-35-binding site, is not responsive to mir-35 (Figure $3 \mathrm{C}-3 \mathrm{~F}$ and $3 \mathrm{O}$, $n>50$ ). When the binding site of mir-35 on lin-23 $3^{\prime}$ UTR was mutated, we found no obvious difference of GFP level between wild type and $g k 262$ harboring the transgenic GS2::GFP ::3'UTRmut ${ }^{\text {lin-23 }}$ (Figure $3 \mathrm{~K}-3 \mathrm{~N}$ and $3 \mathrm{O}, n>50$ ). Therefore, lin-23 is most likely a direct target of mir-35 in G1/S transition regulation.

\section{mir-35 affects germ line proliferation}

Because $g k 262$ has a smaller brood size compared to the wild type, we suspected that mir-35 might play a role in germ line proliferation. Indeed, adult gk262 mitotic region and transition zone were significantly smaller than those of wild type animals (Figure 4A and 4B), while the length of mitotic region remained normal (Figure $4 \mathrm{~F}$ ). There are less mitotic germ cells (about 22\% less nuclei, 
A

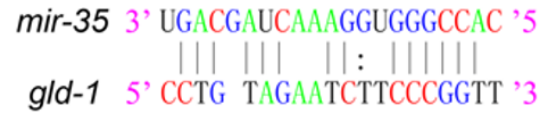

B

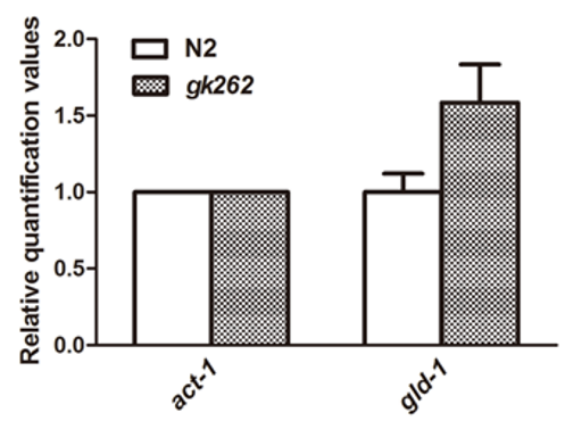

K

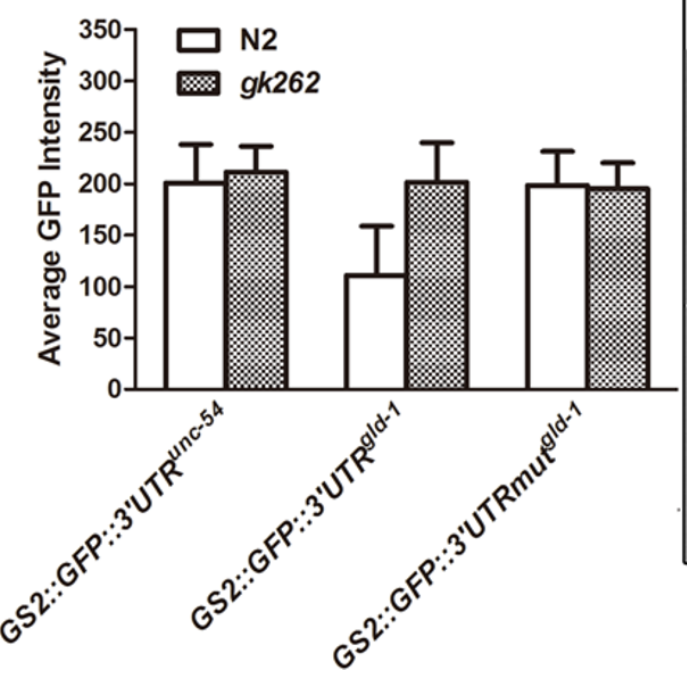

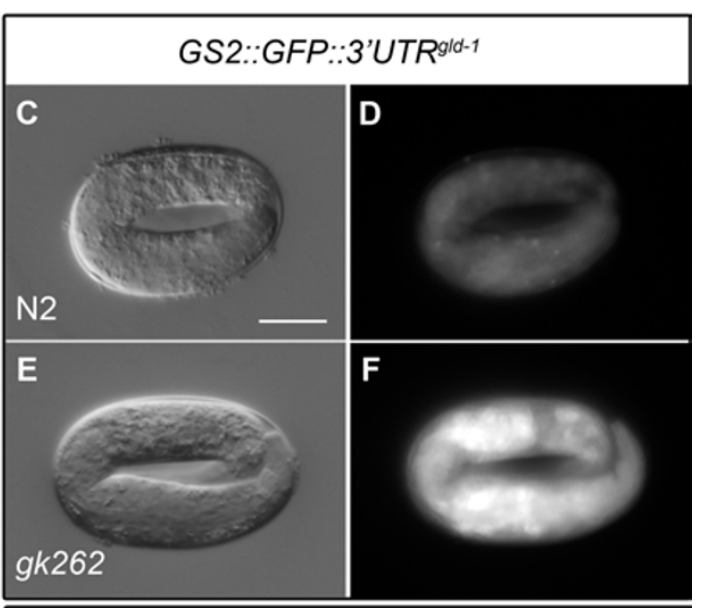

GS2::GFP::3'UTRmutgld-1

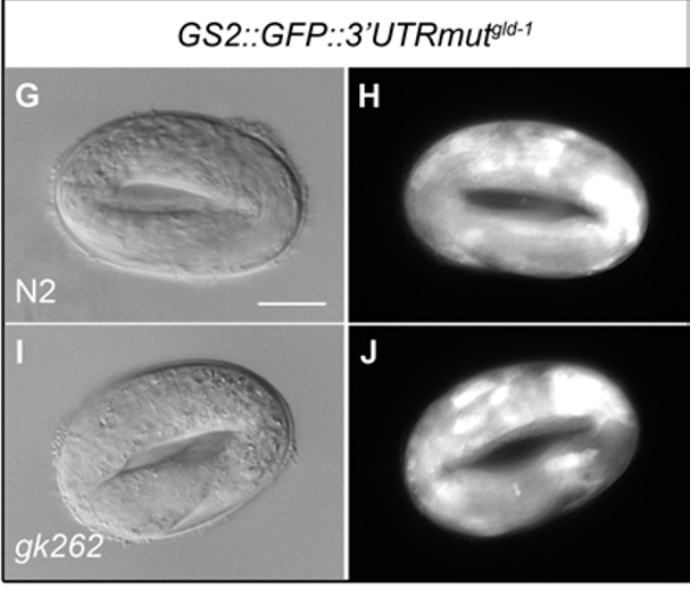

Figure 5 mir-35 may target gld-1. (A) gld-1 is predicted as a target of mir-35 by the bioinformatics program miRanda. Solid lines indicate perfect match, while dash lines indicate non-perfect match. (B) The mRNA level of gld-1 is up regulated in the gk262 at young adult stage revealed by qRT-PCR. (C-J) in vivo GPF reporter experiment shows that gld-1 is a direct target of mir-35. (C-F) A much higher GFP level in gk262 was observed compared to that of the wild type. The DIC (C, E) and the GFP (D, F) pictures are of the same embryos at 2-3 fold stage. (G-J) The $3^{\prime} U T R m u t^{g l d-1}$ has a mutated binding site for mir-35. No obvious difference of GFP level could be observed between wild type and gk262. The DIC (G, I) and the GFP (H, J) pictures are of the same embryos at 2-3 fold stage. The scale bar represents $25 \mu \mathrm{m}$. (K) Quantification of average GFP intensity of the

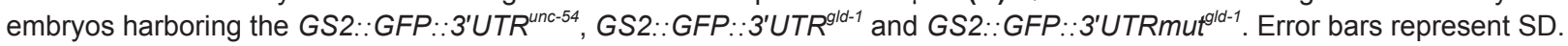

Figure 4F) in the entire mitotic region (Figure 4E).

It appeared that mir-35 might restrict a gonad-specific factor(s) in the mitotic region at the end of the distal gonad arm to regulate a critical germ line proliferation signal. GLD-1 is such a candidate because it is expressed mainly outside of the tip portion of the mitotic region of gonad and inhibits germ line mitosis $[22,24]$. Bioinformatics prediction also revealed that $g l d-1$ may be a direct target of mir-35 (Figure 5A). gld-1(RNAi) led to a larger mitotic region and transition zone (Figure
4A and 4C). Compared to untreated wild type, there were about $19 \%$ more germ cells in the mitotic region of $\operatorname{gld}-1(R N A i)$ animals, while the length of mitotic region increased insignificantly (Figure $4 \mathrm{E}$ and $4 \mathrm{~F}$ ). Furthermore, gld-1(RNAi) suppressed gk262's defects in gonad germ cell number and size of mitotic region (Figure 4B-4F). Noticeably, major changes of the mitotic region normally occurred outside the tip portion of the distal end of gonad arm in $g k 262$ and $g l d-1(R N A i)$ animals (Figure 4A-4D), indicating that mir-35 and gld-1 function mainly 


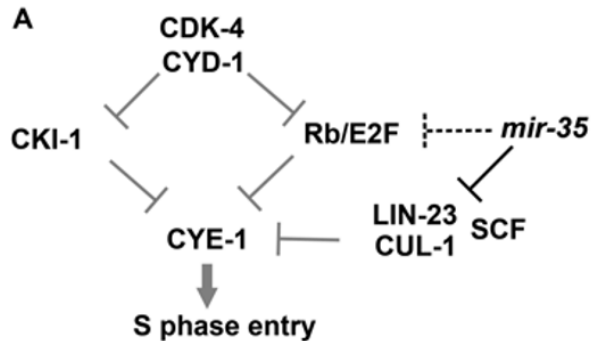

B

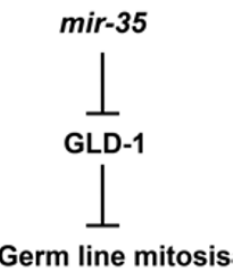

Figure 6 Hypothetical model of mir-35 family function during cell cycle. (A) In the intestinal cells, the mir-35 family suppresses $\mathrm{Rb} / \mathrm{E} 2 \mathrm{~F}$ and SCF pathways to promote the entry of S-phase. lin23 may be the direct target of mir-35 in the SCF pathway, while in the $\mathrm{Rb} / \mathrm{E} 2 \mathrm{~F}$ pathway, the direct target is unknown. (B) In the distal gonad, the mir-35 family targets gld-1 to repress the germ line mitosis.

in germ line proliferation, rather than initial germ cell fate determination and division.

mir-35-41 cluster targets gld-1

To further test whether gld-1 is a mir-35 target gene, we performed quantitative PCR, and found a significantly higher level of gld-1 in gk262 (Figure 5B). We also conducted an in vivo GFP reporter experiment, using the

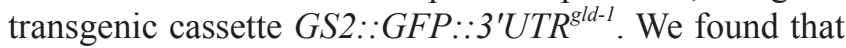
the transgene showed stronger GFP expression in $g k 262$ than in wide type (Figure 5C-5F and $5 \mathrm{~K}, n>50, P<0.001$, Student $t$-test). When we mutated the mir-35-binding site of gld-1 3' UTR, we observed no obvious difference of GFP level between wild type and $g k 262$ harboring the transgenic GS2::GFP::3'UTRmut ${ }^{\text {gld }-1}$ (Figure 5G-5K, n $>50$ ). Taken together, mir-35 targets gld-1 in regulating germ cell proliferation.

\section{Discussion}

We have discovered that the mir-35 family of C. elegans plays an important role in regulating G1/S transition of the cell cycle in intestine and cell proliferation in germ line. In intestinal cells, this regulation is mediated by inhibiting the ubiquitous $\mathrm{Rb} / \mathrm{E} 2 \mathrm{~F}$ pathway and the SCF pathway, in which lin-23 is a direct target of mir-35. In the distal mitotic arm of germ line of hermaphrodites, mir-35 targets gld-1 directly to ensure proper mitotic proliferation of germ cells (Figure 6). Thus, our study reveals how mir-35 participates in the global tune-up of cell cycle/proliferation in the intestine and germ line of $C$. elegans, and indicates that distinct regulatory pathways of cell division/proliferation may be joined by connected miRNAs, in addition to components shared by these pathways.

Alvarez-Saavedra and Horvitz previously showed that without the mir-35 family, most animals undergo developmental arrest at the early embryonic stage and a few show delayed development at normal temperature [14]. During the initial phase of the present study, we suspected that cell cycle defect might be one of the reasons that cause the developmental abnormality, and indeed, we found genetic interactions between mir-35 and $\mathrm{Rb} /$ E2F-related genes. Furthermore, we found a few fertile gk262; lin-36(n766) worms at $25{ }^{\circ} \mathrm{C}$. Additionally, in a pilot EMS-induced mutagenesis suppressor screen, three recovered lines which suppress the intestinal cell cycle defects barely survived at $25{ }^{\circ} \mathrm{C}$ (data not shown). Therefore, the fact that depletion of the mir-35 family leads to cell cycle defect, may at least partially explain the cause(s) of early developmental abnormality of $g k 262$.

In mammalian and cancer cells, several miRNAs have been reported to regulate the G1/S transition of the cell cycle. For instance, miR-17 and miR-20a temper an E2F1-induced G1 checkpoint [47], and miR-195 regulates $\mathrm{G} 1 / \mathrm{S}$ transition [48]. Additionally, miR-18 mediates the degradation of $\beta \operatorname{Tr} C P 1$ (mammalian lin-23 homolog) mRNA, which encodes the main component of the SCF complex [49]. Together with our findings in C. elegans intestine, it appears that posttranscriptional regulation of G1/S transition by miRNAs is well conserved across species. However, other than lin-23 of the SCF pathway, we did not find any target genes of mir-35 in the Rb/E2F pathway, either by bioinformatics prediction or microarray analysis. There are two possible explanations. First, there are target genes of mir-35 yet to be identified in the $\mathrm{Rb} / \mathrm{E} 2 \mathrm{~F}$ pathway. Indeed, by bioinformatics data, over 300 candidate target genes of the mir-35 family have not been studied, or even annotated. Among these genes, some encode proteins that share similar functional domain with that of EFL-1, such as F49E12.6, thus potentially are targets of mir-35-41. The second explanation is that $\mathrm{Rb} / \mathrm{E} 2 \mathrm{~F}$ and SCF pathways might cross-talk so that $\mathrm{Rb} / \mathrm{E} 2 \mathrm{~F}$ could bypass or partially substitute $\mathrm{SCF}$ function in the G1/S transition. This explanation is supported by the experiments showing a synthetic interaction between lin-35 and lin-23 in Rb-regulated cell proliferation [50]. Our experiments presented here indicate that mir-35 participates in adjusting components of both $\mathrm{Rb} / \mathrm{E} 2 \mathrm{~F}$ and SCF pathways, suggesting a cross-talk between the two pathways is necessary for proper cell proliferation. The reduction of proliferating germ cells in $g k 262$ initially suggested to us that both $\mathrm{Rb} / \mathrm{E} 2 \mathrm{~F}$ and SCF pathways may be also negatively regulated by mir-35. However, $\mathrm{Rb}$ is known to be ubiquitously expressed, although the lin-35 mutant does not show abnormal gonad development [51, 
52]. Similarly, lin-23 is expressed in the gonad, but loss of LIN-23 function does not lead to germ cell proliferation defect [36]. Thus, we currently could not exclude that a cross-talk of $\mathrm{Rb} / \mathrm{E} 2 \mathrm{~F}$ and SCF also happens in the gonad.

Because major cell loss was observed in the mitotic region close to the transition zone in $g k 262$ (Figure 4A and 4B), it is highly possible that mir-35 may inhibit a gonad-specific factor(s), which is mainly expressed in the mitotic region and restricts proliferation. It is known that GLP-1/Notch signaling is both necessary and sufficient for germline proliferation at the expense of differentiation, while Gld-1 inhibits germline mitosis and negative feedback regulates GLP-1/Notch signaling [53-55]. In the mitotic region, GLP-1 proteins are present close to where DTC is [56, 57], while GLD-1 is present close to the transition zone $[24,26]$, making gld-1 a top candidate gene responsible for the loss of germ cells in $g k 262$. Indeed, our experiments indicate that mir-35 directly targets $g l d-1$ and a higher than normal level of GLD-1 inhibits germ cell mitosis (Figures 4 and 5). Knock down of GLD-1, in both N2 and gk262, leads to more germ line proliferation in the entire mitotic region, except in the most distal portion. This phenotype is consistent with another aspect of GLD-1 function, i.e., a lower than normal level of GLD-1 promotes germ cell proliferation [58]. The bipartite distribution of GLP-1 and GLD-1 in gonad may also explain why abnormal GLP-1 or GLD-1 level could lead to gonad tumor, a sign of out-of-control mitotic proliferation in hermaphrodites. Interestingly, the mammalian GLD-1 homolog, quaking RNA-binding protein I, is also involved in glioblastoma [59]. We suggest that through modifying GLD-1 expression level and subsequent changes of GLP-1/Notch signaling, mir-35 regulates germline proliferation in the gonad, although the potential involvement of a cross-talk between $\mathrm{Rb} /$ E2F, SCF and GLP-1/Notch pathways is not excluded.

The expression of mir-35-41 is ubiquitous and starts at the onset of gastrulation (our unpublished observation also supports this view). It is therefore not surprising that development of more than one organ is affected when the mir-35-41 cluster is deleted. In addition to intestine and gonad defects, we did observe somatic sex transformation in $g k 262$ hermaphrodites, i.e., increased incidence of ectopic growth of the ray structure (2-fold increase vs wild type) and male-specific neurons (8-fold increase). The fact that the mir-35 family has multiple functions strengthens a notion that miRNAs participate in a wide range of biological processes, likely due to their "one to more" working mode $[5,6,60,61]$. How do mir-3541 achieve organ-specific function? Our experiments presented here show that mir-35-41 target multiple com- ponents of cell proliferation control pathways, to ensure correct cell numbers in the intestine and gonad. By qRTPCR, we also found that mir-35-41 directly or indirectly regulate genes such as fem-2, fem-3 and xol-1, which all encode proteins required in the somatic sex determination pathway [62-65] (our unpublished data). It remains to be seen elusive if such a regulatory scheme of a miRNA family applies to all multi-cell organisms. At least in D. melanogaster, a miRNA cluster is found to impair hedgehog signaling [66].

Precise cell cycle control is essential for genetic stability and accurate cell division of any multi-cell species, employing many if not all conserved regulatory pathways during animal development and differentiation. Although the mir-35-41 family is unique to C. elegans, perhaps representing a newly evolved miRNA species in Caenorhabditis lineage, the target genes, i.e., lin-23 and gld-1, identified in the present study are highly conserved across species. The fact that mammalian cell cycle is regulated by a variety of miRNA species and our present study suggest that targets of miRNAs in cell cycle and proliferation pathways are well conserved in evolution, yet many factors required for the fine regulations remain to be identified. Thus, a systematic search for and research on novel targets of the mir-35 family in $C$. elegans, as exemplified by our study, will further our understanding of cell cycle and proliferation regulation.

\section{Materials and Methods}

\section{Culture conditions and strains}

C. elegans strains were maintained at $20{ }^{\circ} \mathrm{C}$ as described by Brenner [67]. The temperature-sensitive strains were maintained at $20^{\circ} \mathrm{C}$ and in temperature shift assay, synchronized young adults were allowed to lay eggs for $1 \mathrm{~h}$ at $20^{\circ} \mathrm{C}$, and the eggs were shifted to and maintained at $25^{\circ} \mathrm{C}$. For RNAi experiments in the temperature-sensitive strains, we placed dsRNAs injected worms at $20{ }^{\circ} \mathrm{C}$ for $8 \mathrm{~h}$ for RNAi to take effect, shifted injected worms to $25^{\circ} \mathrm{C}$ and obtained 3-fold embryos (RNAi) developed from laid eggs of RNAi animals. Mutations used in this study were as follows: $c d c-25.1(r r 31) I ; \operatorname{rrIs} 1, \operatorname{lin}-35(n 745) I, \operatorname{mir}-35(\mathrm{gk262})$ II, lin-36(n766)III, lin-9(n112)III, efl-1(se1) V, lin-15AB(n309)X. Transgenic markers: rrIs 1 [elt-2::GFP] [39], maIs103[rnr::GFP] [29], gvEx32[cye-1::GFP; rol-6D] were gifts from M Krause.

\section{Nomarski fluorescent microscope examination}

Live animals were mounted to M9 solution in 3\% agar pads, viewed under Zeiss microscopes and imaged by a Zeiss AxioCam.

\section{Rescue gk262 phenotype by microinjection}

To rescue mir-35-41( $g k 262)$, a region (GS1) from 2.16-kb upstream of mir-35 to $1.09-\mathrm{kb}$ downstream of the mir-41 locus, was amplified from genomic DNA with PCR primers (5' ATTCCTCAACCATCGCCT 3' and 5' ATTATCGACGGAGTGCTCG 3'). A region (GS2) was amplified with PCR primers (5' AATAAGCT- 
TATTCCTCAACCATCGCCT 3' and 5' CGGGATCCTAGTTCTAATGGAAAGTACCA 3') and cloned into the plasmid pPD49.78 to replace the $h s p 16.2$ promoter. Individual members of the mir-35-41 cluster were cloned into the modified pPD49.78 with primers listed below:

pre-mir-35 5' CGGGATCCCCCAACTATTATTCTCGGAT 3'; and 5' CGGGTACCTGGAGCAAGTGGAAAAGAT 3', pre-mir-36 5' ACTGGATCCTCTTTTCCACTTGCTCCAC 3' and 5' TATGGTACCATTTTAAGATATTTTATCTTTCCG 3', pre-mir-37 5' ACTGGATCCCCCCGACGCGGAAAGATAA 3' and 5' TATGGTACCCTCACAGAGAAACCACGAGGACC 3', pre-mir-38 5' ACTGGATCCTCGTGGTTTCTCTGTGAGCC $3^{\prime}$ and $5^{\prime}$ TATGGTACCGCTGAATGGTAGAATGTAGGGG 3', pre-mir-39 5' ACTGGATCCTTCTGGAGTTTTCCCCTACATTC 3' and 5' TATGGTACCTGAGGTGCGGACAGGAGG 3', pre-mir-40 5' ACTGGATCCGTGTCTCCGAACCTCCTGTCC $3^{\prime}$ and 5' CATGGTACCCCCACTTAAAAATAACTCACCTTGA 3', pre-mir-41 5' ACTGGATCCCAGCTAAGGTGCGGGTACA 3' and 5' TATGGTACCAGTGCAACGAGTTTTGGACA 3'.

The promoter of ges-1 was amplified with the primers $5^{\prime}$ GCGCATGCTCACCAATACCTTTAGTGAC 3' and 5' GGGGATCCCTGAATTCAAAGATAAGATATGT 3'.

All constructs were injected (co-injected with pRF-4[rol-6(su1006)] at $80 \mathrm{ng} / \mu \mathrm{l})$ to the $g k 262$ at $20 \mathrm{ng} / \mu \mathrm{l}$.

\section{Over-expression of mir-35 and mir-35-41}

To over express mir-35 and mir-35-41, we amplified the premir-35 (primer information is identical to last section) and premir-35-41 (5' CGGGATCCCCCAACTATTATTCTCGGAT 3' and 5' CGGGTACCAACAGCAAAAAAAGAGCAGG 3'). The DNA fragments of pre-mir-35 and pre-mir-35-41 were cloned into the plasmid pPD49.78 containing $h s p 16.2$ promoter. Plasmids were injected (co-injected with pRF-4[rol-6(su1006)] at $80 \mathrm{ng} / \mu \mathrm{l})$ to the $\mathrm{N} 2$ at $20 \mathrm{ng} / \mu \mathrm{l}$. Transgenic embryos between 0 and $3 \mathrm{~h}$ post egg lying, and transgenic animals at arrest L1 and adult stages were heat shocked for $1 \mathrm{~h}$ at $33{ }^{\circ} \mathrm{C}$ to induce over-expression of mir-35 or mir-35-41.

\section{RNAi by microinjection}

Single-strand RNA was transcribed using Promega in vitro transcription kit. The PCR primers are listed below and were amplified from wild-type N2 cDNA:

cki-1 5' GTCGTTGCCTTTTCGGTCGT 3' and 5' CGAGTTCTGATCGTTGGACG 3',

lin-23 5' GTCGTTGCCTTTTCGGTCGT 3' and 5' CGAGTTCTGATCGTTGGACG 3', $d p l-1$ 5' AgtTGGaGgatCTAGTGGAG 3' and 5' GACATAGTGCTGGTGTAAGG 3', $c d c-25.15^{\prime}$ ATCTGGTCGTGTAGCCCTCA 3' and 5' ATGCTGCTTGTCTTGCTTCC 3', lin-9 5' TCTTGCTCTGTCTCCGCCT $3^{\prime}$ and 5' CTTTCGAGCATCTACGTGGC 3',

lin-15B 5' CTTGGCGGTGATTGCTCG 3' and 5' GCACATTGGCTGGTCTCG 3',

gld -1 5'CTCTGAATCGCCATCTCGTT $3^{\prime}$ and 5' TGTTGTTGACTGAAGAAGCC 3'.

The single-stranded RNAs of each gene were annealed to form dsRNA for injection. Embryos from injected animals (24 and $48 \mathrm{~h}$ post microinjection) were collected for further analysis.

\section{DAPI staining}

Approximately 50 mutant animals were sampled on a microscope slide, covered and quickly frozen in liquid nitrogen. After removing cover slip, each slide was placed in methanol, then acetone for 10 minutes each at $-20^{\circ} \mathrm{C}$. After air drying, specimens were treated with DAPI [68] and were ready to be observed.

\section{Gonad dissection}

We performed gonadal dissection essentially as Francis et al. described [53]. Briefly, adult worms were picked onto an unseeded NGM plate, immersed in $2 \mathrm{ml} \mathrm{M} 9$ buffer containing $0.2 \mathrm{mM}$ of levamisole, suspended and transferred to a clean dish. Paralyzed worm was be-headed at level of pharynx with miniature surgical blades, let out the entire gonad arms and transferred with exposed gonads into a $0.5 \mathrm{ml}$ Eppendorf tube for later DAPI staining.

\section{DNA quantification}

To quantify DNA content, nuclei images of DAPI-stained animals were taken with a Zeiss AxioCam and images were analyzed with NIH ImageJ $1.40 \mathrm{~g}$ software. Using ventral nerve cord nuclei as a 2 n DNA standard, $C$ values of intestinal nuclei were estimated by their DAPI-based densitometric quantifications [69, 70].

\section{$R N A$ isolation and quantitative real time PCR}

Synchronized wild-type and mutant animals were kept at $20^{\circ} \mathrm{C}$ until the worms reached L4 larval stage and the synchronized L4 animals were shifted to $25^{\circ} \mathrm{C}$ for $24 \mathrm{~h}$. Total RNA of young adults was extracted using Trizol reagent (Invitrogen). Residual genomic DNA was removed by RNase-free DNase (QIAEN). cDNA was synthesized using random hexamers and SuperScriptII Reverse Transcriptase (Invitrogen). Real-time PCR was performed using SyBR Green PCR Master Mix (Applied Biosystems) on a 7500 Real Time PCR system (Applied Biosystems). Using act-1 as an internal control, relative fold change for transcripts was calculated using comparative $\mathrm{C}_{\mathrm{T}}\left(2^{-\Delta \Delta \mathrm{CT}}\right)$ method. Initial data analysis was carried out using the Applied Biosystems real-time PCR software. Three independent samples were prepared and run in triplicate. Three pairs of primers are listed below:

act-1 5' CCGTGAAAAGATGACCCAAATC 3' and 5' GGTACGTCCGGAAGCGTAGA 3',

lin-23 5' TGTTCCGCCTGCAGTTTGA 3' and 5' GTCCAAAAAGTCCCAAATGAGAA 3',

gld-1 5' GGAAAGTGCTCACCGTGGAA 3' and 5' TGTTCGAGTGCTGCTTGCA 3

\section{GFP reporter assay}

GFP reporters GS2::GFP::3'UTR ${ }^{\text {unc-54 }}$, GS2::GFP:: $3^{\prime} U T R^{\text {lin }}$ ${ }^{23}$, GS2::GFP::3'UTRmut ${ }^{\text {lin-23 }}$ GS2::GFP::3'UTR gld-1 and GS2::GFP::3'UTRmut ${ }^{\text {gld }-1}$ were based on plasmid pPD95.75. GS2 fragment was amplified and cloned into the plasmid pPD95.75, whose original $3^{\prime} U T R^{\text {unc-54 }}$ was replaced with $3^{\prime} U T R^{\text {lin- }}$ ${ }^{23}$ (5' CGCGAATTCATGGCAGTACATTTGATAGT 3' and 5' ATTGGGCCCATGGCAGATTGTGGAGTA 3'), or $3^{\prime} U T R^{\text {gld }-1}$ (5' GGAGAATTCAAAGTGCTCACCGTGGAA 3' and 5' ATAGGGCCCATGGACAGATTGGGAAGG 3'). Using overlap PCR, the mutated $3^{\prime}$ UTR of lin-23 was amplified with two pairs of primers: 5' CCAGAATTCTAGTCAGTTTCTTTTCCG 3' and 5' GGTTTGGTTGATTTCTCGTGGGGTTGAAGATCAGTAAA 3', 5' CACGAGAAATCAACCAAACCCCTTCCCAACTTTTC- 
CTCT $3^{\prime}$ and $5^{\prime}$ TAAGGGCCCATGGCAGATTGTGGAGTAGC $3^{\prime}$. The mutated $3^{\prime}$ UTR of gld -1 was amplified with two pairs of primers: 5' GCGGAATTCTCAGGTCCAGTTTTGATGTC 3' and 5' TTTTTGATGACTACATCTTTGGCGGAAGATTCTACAGG G 3', 5' CAAAGATGTAGTCATCAAAAACCCGCTAGTTAGAT ATGTTC 3' and 5' TTAGGGCCCACAGATTGGGAAGGGG 3'. Reporters were injected (co-injected with pRF-4[rol-6(su1006)] at $80 \mathrm{ng} / \mu \mathrm{l})$ to the $\mathrm{N} 2$ at $20 \mathrm{ng} / \mu \mathrm{l}$.

\section{Acknowledgments}

We thank Caenorhabditis elegans Genetic Center for strains, M Krause of NIDDK, National Institutes of Health for gvEx32 strain, Mei Ding and Xun Huang of Institute of Genetics and Developmental Biology, Chinese Academy of Sciences, and Lei Liu and Liping Wei of School of Life Sciences, Peking University for suggestions and critical comments, and Deming Yang and Ying Gao of our lab for technical help. This project is supported by the National Key Basic Research Program (973 Program) of the Ministry of Science and Technology in China (2007CB946900, 2007CB946904) to Q F, and the National Science Foundation of China (90919034) to D L.

\section{References}

1 Lee RC, Feinbaum RL, Ambros V. The C. elegans heterochronic gene lin-4 encodes small RNAs with antisense complementarity to lin-14. Cell 1993; 75:843-854.

2 Ambros V, Bartel B, Bartel DP, et al. A uniform system for microRNA annotation. RNA 2003; 9:277-279.

3 Lai EC. Micro RNAs are complementary to 3' UTR sequence motifs that mediate negative post-transcriptional regulation. Nat Genet 2002; 30:363-364.

4 Vasudevan S, Tong Y, Steitz JA. Switching from repression to activation: microRNAs can up-regulate translation. Science 2007, 318:1931-1934.

5 Ha I, Wightman B, Ruvkun G. A bulged lin-4/lin-14 RNA duplex is sufficient for Caenorhabditis elegans lin-14 temporal gradient formation. Genes Dev 1996; 10:3041-3050.

6 Moss EG, Lee RC, Ambros V. The cold shock domain protein LIN-28 controls developmental timing in C. elegans and is regulated by the lin-4 RNA. Cell 1997; 88:637-646.

7 Reinhart BJ, Slack FJ, Basson M, et al. The 21-nucleotide let-7 RNA regulates developmental timing in Caenorhabditis elegans. Nature 2000; 403:901-906.

8 Slack FJ, Basson M, Liu Z, Ambros V, Horvitz HR, Ruvkun G. The lin-41 RBCC gene acts in the $C$. elegans heterochronic pathway between the let-7 regulatory RNA and the LIN-29 transcription factor. Mol Cell 2000; 5:659-669.

9 Abbott AL, Alvarez-Saavedra E, Miska EA, et al. The let-7 MicroRNA family members mir-48, mir-84, and mir241 function together to regulate developmental timing in Caenorhabditis elegans. Dev Cell 2005; 9:403-414.

10 Johnston RJ, Hobert O. A microRNA controlling left/right neuronal asymmetry in Caenorhabditis elegans. Nature 2003; 426:845-849.

11 Chang S, Johnston RJ Jr, Frokjaer-Jensen C, Lockery S, Hobert O. MicroRNAs act sequentially and asymmetrically to control chemosensory laterality in the nematode. Nature 2004; 430:785-789.

12 Kato M, Paranjape T, Muller RU, et al. The mir-34 microRNA is required for the DNA damage response in vivo in $C$. elegans and in vitro in human breast cancer cells. Oncogene 2009; 28:2419-2424.

13 Shaw WR, Armisen J, Lehrbach NJ, Miska EA. The conserved miR-51 microRNA family is redundantly required for embryonic development and pharynx attachment in Caenorhabditis elegans. Genetics 2010; 185:897-905.

14 Alvarez-Saavedra E, Horvitz HR. Many families of C. elegans microRNAs are not essential for development or viability. Curr Biol 2010; 20:367-373.

15 Miska EA, Alvarez-Saavedra E, Abbott AL, et al. Most Caenorhabditis elegans microRNAs are individually not essential for development or viability. PLoS Genet 2007; 3:e215.

16 Brenner JL, Jasiewicz KL, Fahley AF, Kemp BJ, Abbott AL. Loss of individual microRNAs causes mutant phenotypes in sensitized genetic backgrounds in C. elegans. Curr Biol 2010; 20:1321-1325

17 Simon DJ, Madison JM, Conery AL, et al. The microRNA $m i R-1$ regulates a MEF-2-dependent retrograde signal at neuromuscular junctions. Cell 2008; 133:903-915.

18 Martinez NJ, Ow MC, Reece-Hoyes JS, Barrasa MI, Ambros VR, Walhout AJ. Genome-scale spatiotemporal analysis of Caenorhabditis elegans microRNA promoter activity. Genome Res 2008; 18:2005-2015.

19 Austin J, Kimble J. $g l p-1$ is required in the germ line for regulation of the decision between mitosis and meiosis in $C$. elegans. Cell 1987; 51:589-599.

20 Berry LW, Westlund B, Schedl T. Germ-line tumor formation caused by activation of $g l p-1$, a Caenorhabditis elegans member of the Notch family of receptors. Development 1997; 124:925-936.

21 Lamont LB, Crittenden SL, Bernstein D, Wickens M, Kimble J. FBF-1 and FBF-2 regulate the size of the mitotic region in the C. elegans germline. Dev Cell 2004; 7:697-707.

22 Suh N, Crittenden SL, Goldstrohm A, et al. FBF and its dual control of gld-1 expression in the Caenorhabditis elegans germline. Genetics 2009; 181:1249-1260.

23 Francis R, Barton MK, Kimble J, Schedl T. gld-1, a tumor suppressor gene required for oocyte development in Caenorhabditis elegans. Genetics 1995; 139:579-606.

24 Jones AR, Francis R, Schedl T. GLD-1, a cytoplasmic protein essential for oocyte differentiation, shows stage- and sexspecific expression during Caenorhabditis elegans germline development. Dev Biol 1996; 180:165-183.

25 Eckmann CR, Crittenden SL, Suh N, Kimble J. GLD-3 and control of the mitosis/meiosis decision in the germline of Caenorhabditis elegans. Genetics 2004; 168:147-160.

26 Suh N, Jedamzik B, Eckmann CR, Wickens M, Kimble J. The GLD-2 poly(A) polymerase activates gld-1 mRNA in the Caenorhabditis elegans germ line. Proc Natl Acad Sci USA 2006; 103:15108-15112.

27 Schmid M, Kuchler B, Eckmann CR. Two conserved regulatory cytoplasmic poly(A) polymerases, GLD-4 and GLD-2, regulate meiotic progression in C. elegans. Genes Dev 2009; 23:824-836.

28 Hedgecock EM, White JG. Polyploid tissues in the nematode 
Caenorhabditis elegans. Dev Biol 1985; 107:128-133.

29 Hong Y, Roy R, Ambros V. Developmental regulation of a cyclin-dependent kinase inhibitor controls postembryonic cell cycle progression in Caenorhabditis elegans. Development 1998; 125:3585-3597.

30 Feng H, Zhong W, Punkosdy G, et al. CUL-2 is required for the G1-to-S-phase transition and mitotic chromosome condensation in Caenorhabditis elegans. Nat Cell Biol 1999; 1:486-492.

31 Fukuyama M, Gendreau SB, Derry WB, Rothman JH. Essential embryonic roles of the CKI-1 cyclin-dependent kinase inhibitor in cell-cycle exit and morphogenesis in C. elegans. Dev Biol 2003; 260:273-286.

32 Dealy MJ, Nguyen KV, Lo J, et al. Loss of Cull results in early embryonic lethality and dysregulation of cyclin E. Nat Genet 1999; 23:245-248.

33 Wang Y, Penfold S, Tang X, et al. Deletion of the Cull gene in mice causes arrest in early embryogenesis and accumulation of cyclin E. Curr Biol 1999; 9:1191-1194.

34 Hebeisen M, Roy R. CDC-25.1 stability is regulated by distinct domains to restrict cell division during embryogenesis in C. elegans. Development 2008; 135:1259-1269.

35 Kipreos ET, Lander LE, Wing JP, et al. cul-1 is required for cell cycle exit in C. elegans and identifies a novel gene family. Cell 1996; 85:829-839.

36 Kipreos ET, Gohel SP, Hedgecock EM. The C. elegans F-box/ WD-repeat protein LIN-23 functions to limit cell division during development. Development 2000; 127:5071-5082.

37 Ashcroft N, Golden A. CDC-25.1 regulates germline proliferation in Caenorhabditis elegans. Genesis 2002; 33:1-7.

38 Kim J, Lee AR, Kawasaki I, Strome S, Shim YH. A mutation of $c d c$-25.1 causes defects in germ cells but not in somatic tissues in C. elegans. Mol Cells 2009; 28:43-48.

39 Kostic I, Roy R. Organ-specific cell division abnormalities caused by mutation in a general cell cycle regulator in C. elegans. Development 2002; 129:2155-2165.

40 Colavita A, Culotti JG. Suppressors of ectopic UNC-5 growth cone steering identify eight genes involved in axon guidance in Caenorhabditis elegans. Dev Biol 1998; 194:72-85.

41 Thomas JH, Horvitz HR. The C. elegans gene lin-36 acts cell autonomously in the lin-35/Rb pathway. Development 1999; 126:3449-3459.

42 Ceol CJ, Horvitz HR. $d p l-1$ DP and efl-1 E2F act with lin-35/ $\mathrm{Rb}$ to antagonize Ras signaling in C. elegans vulval development. Mol Cell 2001; 7:461-473.

43 Boxem M, van den Heuvel S. lin-35 Rb and cki-1 Cip/Kip cooperate in developmental regulation of G1 progression in $C$. elegans. Development 2001; 128:4349-4359.

44 Boxem M, van den Heuvel S. C. elegans class B synthetic multivulva genes act in G(1) regulation. Curr Biol 2002; 12:906-911.

45 Page BD, Guedes S, Waring D, Priess JR. The C. elegans $\mathrm{E} 2 \mathrm{~F}$ - and DP-related proteins are required for embryonic asymmetry and negatively regulate Ras/MAPK signaling. Mol Cell 2001; 7:451-460.

46 Beitel GJ, Lambie EJ, Horvitz HR. The C. elegans gene lin-9, which acts in an $\mathrm{Rb}$-related pathway, is required for gonadal sheath cell development and encodes a novel protein. Gene 2000; 254:253-263.
47 Pickering MT, Stadler BM, Kowalik TF. miR-17 and miR-20a temper an E2F1-induced G1 checkpoint to regulate cell cycle progression. Oncogene 2009; 28:140-145.

48 Xu T, Zhu Y, Xiong Y, Ge YY, Yun JP, Zhuang SM. MicroRNA-195 suppresses tumorigenicity and regulates G1/S transition of human hepatocellular carcinoma cells. Hepatology 2009; 50:113-121.

49 Elcheva I, Goswami S, Noubissi FK, Spiegelman VS. CRD$\mathrm{BP}$ protects the coding region of betaTrCP1 mRNA from miR183-mediated degradation. Mol Cell 2009; 35:240-246.

50 Fay DS, Keenan S, Han M. fzr-l and lin-35/Rb function redundantly to control cell proliferation in C. elegans as revealed by a nonbiased synthetic screen. Genes Dev 2002; 16:503-517.

51 Bender AM, Kirienko NV, Olson SK, Esko JD, Fay DS. lin$35 / \mathrm{Rb}$ and the CoREST ortholog spr-1 coordinately regulate vulval morphogenesis and gonad development in C. elegans. Dev Biol 2007; 302:448-462.

52 Bender AM, Wells O, Fay DS. lin-35/Rb and $x n p-1 /$ ATR-X function redundantly to control somatic gonad development in C. elegans. Dev Biol 2004; 273:335-349.

53 Francis R, Barton MK, Kimble J, Schedl T. gld-1, a tumor suppressor gene required for oocyte development in Caenorhabditis elegans. Genetics 1995; 139:579-606.

54 Hansen D, Schedl T. The regulatory network controlling the proliferation-meiotic entry decision in the Caenorhabditis elegans germ line. Curr Top Dev Biol 2006; 76:185-215.

55 Marin VA, Evans TC. Translational repression of a C. elegans Notch mRNA by the STAR/KH domain protein GLD-1. Development 2003; 130:2623-2632.

56 Crittenden SL, Bernstein DS, Bachorik JL, et al. A conserved RNA-binding protein controls germline stem cells in Caenorhabditis elegans. Nature 2002; 417:660-663.

57 Crittenden SL, Troemel ER, Evans TC, Kimble J. GLP-1 is localized to the mitotic region of the $C$. elegans germ line. Development 1994; 120:2901-2911.

58 Hansen D, Wilson-Berry L, Dang T, Schedl T. Control of the proliferation versus meiotic development decision in the $C$. elegans germline through regulation of GLD-1 protein accumulation. Development 2004; 131:93-104.

59 Chenard CA, Richard S. New implications for the QUAKING RNA binding protein in human disease. J Neurosci Res 2008; 86:233-242.

60 Boehm M, Slack F. A developmental timing microRNA and its target regulate life span in C. elegans. Science 2005; 310:1954-1957.

61 Li X, Cassidy JJ, Reinke CA, Fischboeck S, Carthew RW. A microRNA imparts robustness against environmental fluctuation during development. Cell 2009; 137:273-282.

62 Chin-Sang ID, Spence AM. Caenorhabditis elegans sexdetermining protein FEM-2 is a protein phosphatase that promotes male development and interacts directly with FEM-3. Genes Dev 1996; 10:2314-2325.

63 Hodgkin J. A genetic analysis of the sex-determining gene, tra-1, in the nematode Caenorhabditis elegans. Genes Dev 1987; 1:731-745.

64 Mehra A, Gaudet J, Heck L, Kuwabara PE, Spence AM. Negative regulation of male development in Caenorhabditis elegans by a protein-protein interaction between TRA-2A and 
FEM-3. Genes Dev 1999; 13:1453-1463.

65 Miller LM, Plenefisch JD, Casson LP, Meyer BJ. xol-1: a gene that controls the male modes of both sex determination and X chromosome dosage compensation in C. elegans. Cell 1988; 55:167-183.

66 Friggi-Grelin F, Lavenant-Staccini L, Therond P. Control of antagonistic components of the hedgehog signaling pathway by microRNAs in Drosophila. Genetics 2008; 179:429-439.

67 Brenner S. The genetics of Caenorhabditis elegans. Genetics 1974; 77:71-94.
68 Miller DM, Shakes DC. Immunofluorescence microscopy. Methods Cell Biol 1995; 48:365-394.

69 Boxem M, Srinivasan DG, van den Heuvel S. The Caenorhabditis elegans gene $n c c-1$ encodes a $c d c 2$-related kinase required for $\mathrm{M}$ phase in meiotic and mitotic cell divisions, but not for S phase. Development 1999; 126:22272239.

70 Lozano E, Saez AG, Flemming AJ, Cunha A, Leroi AM. Regulation of growth by ploidy in Caenorhabditis elegans. Curr Biol 2006; 16:493-498. 